psychiatry, and promotes psychiatry as a career choice. This survey has been helpful in identifying what aspects of the scheme are particularly attractive, and also, importantly, how the Psych Stars scheme can be improved for future cohorts. The survey will be delivered to all future annual cohorts of Psych Stars and mentors.

\section{Digitalised remote-delivery of AVATr Simulation in Psychiatry: a unique success in COVID-19 pandemic \\ Vimal Mannali $^{1 \star}$, Paul Strickland ${ }^{2}$, Craig laBuscagne ${ }^{1}$ and Joy Clift ${ }^{1}$ \\ ${ }^{1}$ Surrey and Borders Partnership NHS Foundation Trust and \\ ${ }^{2}$ Xenodu Virtual Environments \\ ${ }^{\star}$ Corresponding author.}

\section{doi: 10.1192/bjo.2021.411}

Aims. Surrey and Borders NHS Foundation Trust's AVATr (Augmented Virtual-reality Avatar in Training) is a unique ground-breaking Virtual Patient Simulation System, which uses the Xenodu platform to train learners in essential clinical and complex communication skills. Over 30 patient scenarios have been developed after identifying learner-specific development needs, including exploration of overt psychosis, assessment of capacity, sharing bad news, and neglect in care home residents. Actors are filmed responding to several domains of clinical questions, further categorised into three narrative-modes of being 'Engaged, Neutral or Disengaged', to build a bank of scenarios. During the session, the trainee is projected on to a large screen, using a camera and video special effects, which results in a life-like interaction with the Virtual Patient. Trainees can view themselves interacting with the Virtual Patient in real-time, from a unique 'out-of-body' perspective, immersed in a custom-designed interactive virtual environment. The simulation facilitator engages with the learner and determines the appropriate choices of responses for the Virtual Patient and if needed, can prompt with explorative cues to continue the narrative-linked conversation. AVATr model pioneered in United Kingdom the use of an innovative 'self-observational approach' in Psychiatry training. This is different to a first-person perspective used in virtual or augmented-reality systems in several clinical specialties. The use of Facilitated-Debrief and Peer-Debrief in sessions, render another layer to the simulation experience.

Method. During the COVID-19 pandemic, we evolved the AVATr model to remote or hybrid sessions, where simulations were digitally enhanced, and have been run through Microsoft Teams. The simulation facilitator is connected to a multi-user video call, enabling the Virtual Patient to be projected as an attendee using Microsoft Teams.

Result. The hybrid model of AVATr has received tremendous feedback, as it now simulates video-consultations that a vast majority of Psychiatry trainees, especially community-based, undertake due to COVID-19 restrictions. The format of AVATr simulation sessions has remained unchanged, and the remote delivery has been particularly successful as it allows trainees to log in from different remote locations to come together for an interactive training session, without any physical restrictions.

Conclusion. Since 2015, our simulation platform has been utilised for Post-Graduate Medical Education, to enhance essential professional skills and stimulate professional growth. Currently the hybrid model of AVATr is being expanded to Nursing, Psychology and Allied Health Professional (AHP) clinical training streams, along with Undergraduate Medical Education, to address identified gaps in face-to-face training amidst COVID-19 pandemic.
A cross-sectional study exploring attitudes of first year medical students towards psychiatry and factors they identified that would help stimulate their interest

Isabel Mark* and Victoria Fernandez Garcia De Las Heras

South West London and St George's Mental Health Trust, St George's University of London

${ }^{\star}$ Corresponding author.

doi: 10.1192/bjo.2021.412

Aims. Psychiatry has had long standing recruitment difficulties. Many efforts have been made to explore strategies that encourage interest in the specialty, with early university experience being an important factor in ultimate career choice. The Royal College of Psychiatrists 'Choose Psychiatry' guidance for medical schools outlines four key areas of focus: teaching excellence, placement quality, leadership and enrichment activities, with other research reporting similar conclusions. The aim of this study was to assess attitudes towards psychiatry amongst first year medical students, examine what input they would welcome from psychiatrists at this stage of their career and consider if their wishes are in keeping with the 'Choose Psychiatry' guidance.

Method. All first-year medical students at St George's University of London were approached in October 2019 and offered the opportunity for early psychiatry exposure. 60 students were recruited. Data were collected in November 2019 via an online questionnaire comprising of baseline demographics, the 30-item Attitudes Towards Psychiatry questionnaire (ATP-30) and a freetext question asking what students would like from psychiatry at this stage of their education. Quantitative data were analysed using Excel, whilst qualitative data were analysed thematically.

Result. The mean ATP-30 score was 113.83 (SD 12.57, range 70-135). Gender, ethnicity and religious background were not associated with a change in ATP score. Undergraduates' attitudes were more positive than those of postgraduates (independent $t$-test revealed a p-value of 0.087 ). Seven themes were identified outlining what students wanted from psychiatry, the most prominent being: (a) learning about the lifestyle of a psychiatrist and finding a role model, (b) exploring the patient perspective, (c) exploring the interaction between psychiatry and specialities and (d) having an opportunity to develop communication skills.

Conclusion. The findings demonstrate higher ATP-30 results than previous literature has reported, potentially due to mental health awareness campaigns in recent years. As undergraduates were found to have a higher mean score, targeting them for additional psychiatry contact may be beneficial. Themes identified by students in this study support 'Choose Psychiatry' guidance, whilst also highlighting the potential for combining some psychiatry with other specialties in the curriculum. Integration with communication skills teaching might help engage those not initially psychiatry-inclined. Further study will be required to establish whether implementing the suggested strategies can lead to improvement in student ATP-30 scores and ultimately increased recruitment rates.

Increasing staff confidence about domestic abuse identification, disclosure and safeguarding in a community mental health team

Beth McCausland ${ }^{1 *}$, Nicola Minicozzi ${ }^{1}$, Siobhan O'Halloran ${ }^{1}$, Avril Ward ${ }^{2}$ and Kerry Elliott ${ }^{3}$

${ }^{1}$ Southern Health NHS Foundation Trust, University of Southampton; ${ }^{2}$ Yellow Door, Pathfinder and ${ }^{3}$ Aurora New Dawn Ltd, Pathfinder

${ }^{\star}$ Corresponding author.

doi: 10.1192/bjo.2021.413 
Aims. To increase staff confidence about identifying Domestic Abuse (DA), particularly regarding 'how to ask' to encourage disclosure and the pathways available for appropriately safeguarding survivors; in a Community Mental Health Team (CMHT) setting. Background. DA is bi-directionally associated with mental health $(\mathrm{MH})$ disorders; 1:4 women in contact with $\mathrm{MH}$ services are currently experiencing DA. MH professionals (MHPs) are in a privileged position to identify DA and support survivors. However, this is dependent on MHPs receiving adequate training about DA. For this, we collaborated with Pathfinder, a national pilot project run by a consortium of five expert partners that aims to establish comprehensive health practice in relation to DA and Violence Against Women \& Girls in Acute Hospital Trusts, MH Trusts and Primary Care. In Southampton, Pathfinder has funded two domestic and sexual abuse (DSA) advocates to both train $\mathrm{MH}$ staff and take a small caseload of $\mathrm{MH}$ service users who are experiencing abuse.

Method. We conducted a baseline survey of staff confidence across the following domains:

Knowing the legal definition of DA,

The process used to escalate a DA concern,

How to make a referral,

How to complete DASH forms,

How and when to refer to Pathfinder,

What the following acronyms mean: PIPPA, MAPPA, MARAC, IDVA, DASH,

What HRDA and MASH mean,

How to ask about DA,

Who to signpost service users to if they make a disclosure, and when to involve the police.

We presented the survey results at the regional Pathfinder strategic group, with Trust management representatives present. This project fits within the strategic group's sustainability aims to increase DA awareness and safeguarding processes across the Trust.

The Pathfinder funded DSA Advisors delivered a four-hour training package targeting the surveyed questions and wider information on DA. We then re-surveyed to see if staff confidence had increased. We are currently analyzing the number of referrals to the Pathfinder service pre- and post-training.

Result. Staff confidence increased across all domains following the training (\% mean increase): Qs1 (35\%), Qs2 (9\%), Qs3 (45\%), Qs4 (81\%), Qs5 (25\%), Qs6 (49\%), Qs7 (89\%), Qs8 (62\%) and Qs9 (48\%).

We have now arranged a bi-monthly drop-in at the CMHT by the DSA advisor who provided the training, to embed the link between the services and maintain staff confidence. We will circulate these results to advocate that this training is provided across the Trust.

Physical Health Simulation Based Education (SBE) for psychiatrists in the first COVID-19 wave: improving the competence and confidence of the medical workforce

Craig McEwan*, Richard Kerslake and Michael Hobkirk

Sussex Partnership NHS Foundation Trust (SPFT)

${ }^{*}$ Corresponding author.

doi: 10.1192/bjo.2021.414

Aims. At the start of the COVID-19 pandemic there was significant uncertainty for the NHS and it's workforce. Within psychiatry, there was an expectation that junior doctors would be redeployed, with senior psychiatrists stepping down to cover physical health and on-call duties.

Senior leadership in mental health trusts were also preparing for COVID-19 outbreaks on psychiatric wards and were developing strategies for managing a novel illness with a poorly understood clinical course. Many psychiatrist expressed anxieties around their competency in assessing and managing acutely physically unwell patients in a mental health setting.

This project aimed to improve confidence of psychiatrists in core physical health competencies through devising and delivering an evolving SBE package.

Method. Sussex Partnership Foundation Trust redeployed two higher trainees from their simulation faculty to work full time on developing a SBE package. This was requested by senior leadership to deliver training about assessing and managing physically unwell patients in the context of COVID-19. This training was devised as a 90 minute didactic lecture following by 90 minutes of SBE.

This was delivered at 6 sites through 10 opt-in sessions available to all doctors in the trust over 4 weeks. Pre and post-course questionnaires were given to all participants to measure the effect. Result. 102 medical staff attended the SBE workshops. Feedback was completed by $93(91 \%)$ doctors prior to the course and 97 (95\%) post. Before the workshop, 33\% did not feel they had a structured approach for assessing an acutely unwell patient, which reduced to $0 \%$ after completing the course.

On a 5-point Likert scale, confidence in managing COVID-19 symptoms increased from $2.54 / 5$ to $4.07 / 5$ overall with $89 \%$ of doctors feeling "confident" or "very confident". There were similar increases in confidence in managing critically unwell patients (2.7/5 pre; $3.95 / 5$ post) and in identifying alternative causes for acutely unwell patients (2.63/5 pre; $4.02 / 5$ post).

Conclusion. This project demonstrates that SBE is an effective way to rapidly develop effective interventions for the medical workforce, increasing confidence in the face of significant uncertainty and reducing anxiety within the system to meet the learning needs identified by medical leadership.

As part of this project Sussex Partnership Medical Education freely shared the workshop materials, which were later adopted and used by psychiatry departments internationally.

\section{Responding to a pandemic with Simulation Based} Education (SBE)? Sharing lessons learned from Sussex Partnership Foundation Trust (SPFT)

Craig McEwan*, Richard Kerslake and Michael Hobkirk

Sussex Partnership NHS Foundation Trust (SPFT)

${ }^{\star}$ Corresponding author.

doi: 10.1192/bjo.2021.415

Aims. In March 2020 SPFT was preparing for the first wave of the COVID-19 pandemic. Senior medical leadership supported the rapid development and delivery of SBE workshop for assessment and management of physically unwell patients in a psychiatric setting in the context of COVID-19. The training was delivered to 102 psychiatrists across 10 sessions over 4 weeks.

A learning review was completed to identify lessons learned from the delivery of this SBE workshop.

Method. The intervention was reviewed using open-space feedback from attendees, interviews with facilitators and medical leadership, and SWOT (Strengths, Weaknesses, Opportunities, Threats) analysis. 